\title{
Sauber macht krank - Das Zusammenspiel von Händehygiene und Handekzemen
}

\section{Cleanliness Turns into Illness - The Interconnection of Handhygiene and Hand Excema}

\author{
Autor \\ H. Löffler \\ Institut \\ Hautklinik der SLK-Kliniken Heilbronn, Akademisches Lehrkrankenhaus der Universität Heidelberg
}

\section{Bibliografie}

DOI $10.1055 / \mathrm{s}-2008-1077610$

Akt Dermatol 2008; 34:

371-375 @ Georg Thieme

Verlag KG Stuttgart · New York ISSN 0340-2541

Korrespondenzadresse

Prof. Dr. Harald Löffler

Hautklinik

SLK-Kliniken

Am Gesundbrunnen 20-24

74078 Heilbronn

Harald.Loeffler@slk-kliniken.de

\section{Zusammenfassung \\ $\nabla$}

Für Mitarbeiter in medizinischen Berufen gehören irritative Hautveränderungen an den Händen sowie Handekzeme zum Alltag. Ursächlich sind nicht zuletzt Maßnahmen der Händehygiene. Von vielen Mitarbeitern im Pflegeberuf werden insbesondere die alkoholischen Händedesinfektionsmittel für ihre rauen Hände verantwortlich gemacht, nicht selten werden sogar Allergien gegen die Alkohole vermutet. In ausführlichen allergologischen Testungen konnte diese Vermutung widerlegt werden - Sensibilisierungen gegen die Alkohole wurden beim medizinischen Personal bislang nicht beobachtet, falls allergische Reaktionen vorkamen, dann gegen andere Inhaltsstoffe der Desinfektionsmittel. Auch in den Irritationstestungen konnte gezeigt werden,

Händehygienemaßnahmen zählen zu den Kernprozeduren im medizinischen Alltag. Ihre korrekte Durchführung ist essenziell für die Vermeidung von nosokomialen Infektionen und die Verbreitung von multiresistenten Erregern [1]. Leider bleiben die notwendigen Hygienemaßnahmen zum Teil erheblich unter den notwendigen und empfohlenen Maßnahmen [2], nicht zuletzt weil die Hautverträglichkeit der Produkte von den Betroffenen als sehr problematisch eingeschätzt wird.

\section{Epidemiologische Daten \\ $\nabla$}

Hautveränderungen an den Händen gehören für viele Mitarbeiter des Gesundheitsdienstes zum Alltag. Ausgetrocknete Hände und eine raue schuppige Haut wird als „zum Beruf gehörend“ hingenommen und kaum hinterfragt. Bei Reihen- dass die alkoholischen Händedesinfektionsmittel in aller Regel gut vertragen werden, zumindest deutlich besser als die Händewaschung mit Detergentien. Der Grund, warum viele Pflegedienstmitarbeiter so negativ gegen die Händedesinfektion mit alkoholischen Produkten eingestellt sind, ist, dass die Präparate auf vorgeschädigter Haut ein brennendes Gefühl verursachen. Dieses Gefühl führt dazu, dass die alkoholische Desinfektion zugunsten des Händewaschens vernachlässigt wird, was dann wieder den Hautzustand weiter verschlechtert. Eine rechtzeitige Aufklärung der Mitarbeiter im Gesundheitsdienst über die Hautverträglichkeit von Händedesinfektionsmaßnahmen sollte bereits bei der Ausbildung erfolgen, um von Anfang an eine Schonung der Haut zu gewährleisten.

untersuchungen von medizinischem Personal (insbesondere der Pflegeberufe) wurden Prävalenzwerte von Kontaktdermatitiden der Hände von 20 bis $40 \%$ gefunden [3-6]. Irritative Veränderungen stehen dabei in der Häufigkeit vor allergischen oder atopischen Manifestationen. Die Krankenpflege und verwandte Gebiete zählten mit einem 6-fach erhöhten Erkrankungsrisiko zu den Risikoberufen für den Erwerb von Berufsdermatosen [5,7], die Berufsgruppe des Pflegepersonals und der Arzthelferinnen steht in Deutschland auf Platz 5 einer Hitliste für Berufsdermatosen, zumeist wegen irritativer Ekzemerkrankungen [8-10]. Die vielfach leichten Veränderungen (trockene Haut der Hände sowie interdigitale diskrete Ekzeme) werden häufig nicht ernst genommen. Ein folgenschwerer Fehler, da gerade diese geringen Veränderungen ein Marker für künftige schwere Handekzeme sein können [11]. 


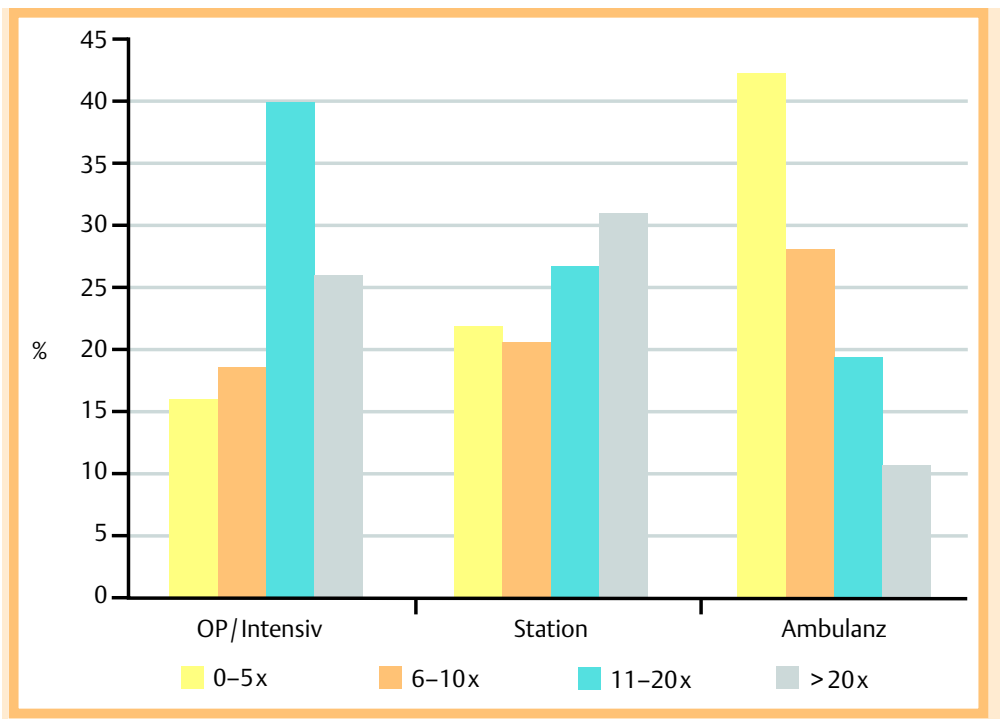

Abb. 1 Häufigkeit der Händedesinfektion pro Schicht.

\section{Einschåtzung der Pathogenese \\ $\nabla$}

Doch woran liegen diese Hautveränderungen? Die Mitarbeiter im Gesundheitsdienst führen Handlungen durch, welche als Risikohandlungen für Handekzeme angesehen werden können. Im Vordergrund steht hierbei häufig die Händehygiene [8]. Seit den Zeiten von Ignaz Semmelweis hat sich die Händehygiene als eine der Kernprozeduren im Gesundheitsdienst durchgesetzt [12]. Leider sind viele dieser Maßnahmen der Händehygiene mit der Gefahr von Hautschäden verbunden, wobei die Einschätzung bezüglich der Toxizität der einzelnen Maßnahmen sehr unterschiedlich ist [13-15]. Um zu untersuchen wie die Pflegekräfte selbst die verwendeten Prozeduren einschätzen, wurde durch die Deutsche Kontaktallergiegruppe eine Fragebogenaktion an mehreren deutschen Universitätskliniken initiiert [16]. Hier zeigte sich eine unterschiedliche Verteilung der Häufigkeit von Händehygienemaßnahmen, je nachdem wo die Pflegekraft eingesetzt wurde. Am häufigsten wurden Hygieneprozeduren (wie die Desinfektion) auf Intensivstationen und im OP durchgeführt, am seltensten in den Ambulanzen ( $\bullet$ Abb. 1). Es zeigte sich, dass über 70\% der Pflegekräfte über irritative Hautveränderungen innerhalb des letzten Jahres berichteten. Das Auftreten dieser Hautveränderungen war dabei im Ambulanzbereich am geringsten. Besonders eindrücklich war das Ergebnis bezüglich der Einschätzung der Toxizität. Hier glaubten fast 70\% der Pflegekräfte dass die alkoholische Händedesinfektion für ihre Hände schädlicher sei als das Waschen. Über $15 \%$ aller Pflegedienstmitarbeiter glaubten darüber hinaus, dass eine allergische Reaktion gegen Alkohole bei ihnen vorläge. In der sich anschließenden allergologischen Testung konnte jedoch bei keinem einzigen der Getesteten eine Sensibilisierung gegenüber einem Alkohol festgestellt werden. Bei zwei Testungen gab es positive Testergebnisse, die reinen Alkohole waren dabei jedoch nie die Ursache (vielmehr andere Inhaltsstoffe wie z.B. der Emulgator Cetearyl Octanoat). Vergleicht man diese Untersuchungsergebnisse (und der fehlenden Beschreibung eines allergischen Kontaktekzems bei einem Mitarbeiter des Gesundheitsdienstes auf einen Alkohol in der Literatur) mit der unglaublichen Menge von über 10 Tonnen alkoholische Händedesinfektionsmittel, welche täglich allein in Deutschland auf die Hände appliziert werden (ca. 7,1 Liter pro Bett und Jahr) [17], so stellt sich die Frage, ob es überhaupt Allergien gegen die in der Händehygiene verwendeten Alkohole gibt.
Am ehesten dürften die wenigen kutanen Allergien auf Alkohole wie Ethanol auf die Akkumulation von Acetaldehyd zurückzuführen sein, welche bei einer Defizienz der Aldehydreduktase auftreten kann $[18,19]$.

\section{Schädigung durch alkohol-basierte Hände- desinfektionsmittel und durch Waschung $\nabla$}

Trotz der fehlenden Sensibilisierungen berichten viele Pflegekräfte über Probleme durch die alkoholischen Händedesinfektionsmittel, welche zu deren verminderten Einsatz führen. Sind die alkoholischen Händedesinfektionsmittel tatsächlich schädlich für die Haut, wie dieses von den Pflegedienstmitarbeitern angenommen wird? Diesbezüglich führten wir eine repetitive Irritationstestung mit Ethanol, Propanol und Isopropanol in den unterschiedlichsten Konzentrationen durch [20]. Es fand sich nach 48-stündiger Applikation keine allergische Reaktion und nur eine geringe Erhöhung des transepidermalen Wasserverlustes (als Marker der epidermalen Barriere) in gleicher Höhe wie nach Applikation von Wasser oder einer Leerkammer. Bei der ebenfalls durchgeführten Messung der kutanen Hydratation konnte eine leichte Verminderung der Hautfeuchte durch die Alkohole festgestellt werden [20].

Von größerer Relevanz dürfte jedoch die standardisierte Irritations-Testung in Form eines repetitiven Applikationstests sein. Hierbei wurde über 1 Woche mit einem Schaumstoffroller mehrfach täglich sowohl die alkoholische Händedesinfektion (80\%ig Ethanol) als auch eine Händewaschung mit dem anionischen Detergens (Natriumlaurylsulfat 0,5\%) simuliert [20]. Das Ergebnis zeigte hierbei, dass die standardisierte Händewaschung eine sehr viel stärkere Schädigung der epidermalen Barriere (gemessen als Anstieg des transepidermalen Wasserverlustes), aber auch eine deutlich stärkere Austrocknung der Haut, als die 80\%ige Ethanollösung hervorrief. Häufig wird jedoch gerade im OP die Kombination aus Waschung und Desinfektion durchgeführt. Im Folgenden untersuchten wir daher die Kombinationsanwendung des Händewaschens mit der alkoholischen Händedesinfektion. Erstaunlicherweise konnten wir feststellen, dass die Kombination aus Händewaschung und Desinfektion zu einer geringeren irritativen Schädigung (gemessen an der Barriereveränderung sowie an der Veränderung an der kutanen Hydrata- 


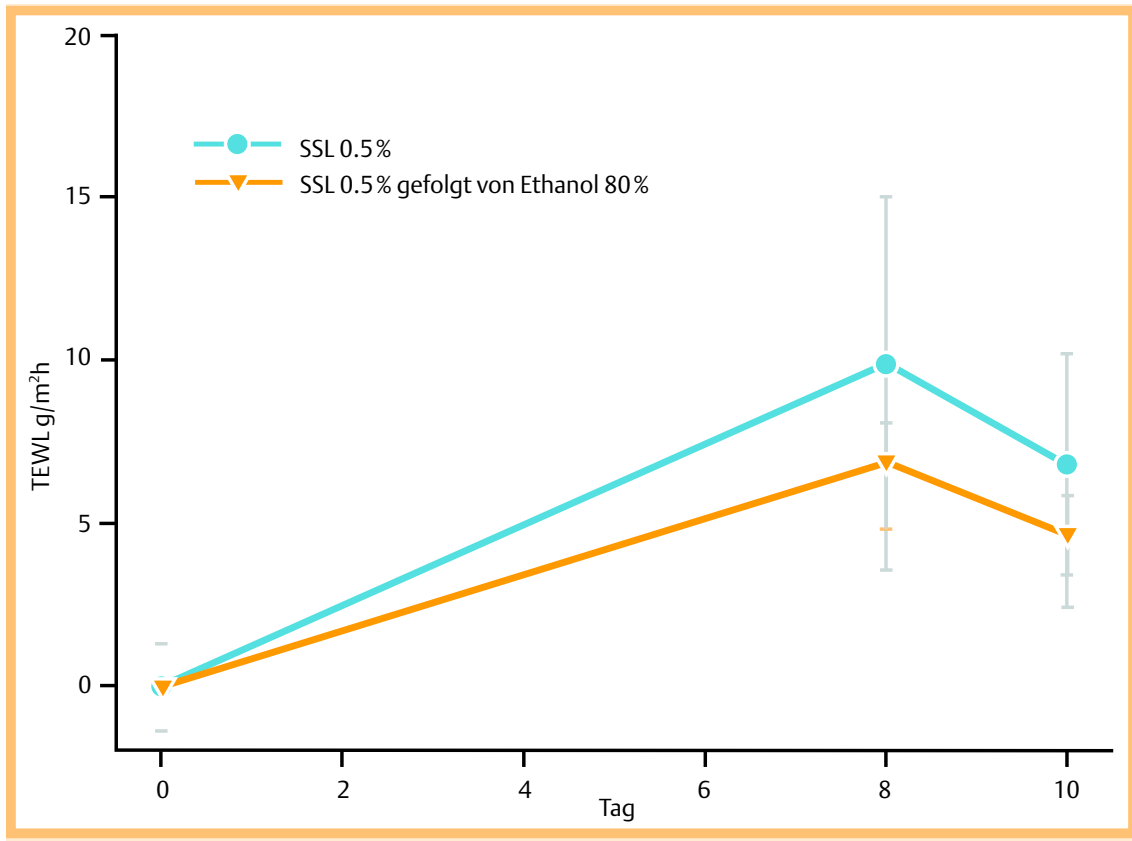

Abb. 2 Reduzierte Irritation gemessen am transepidermalen Wasserverlust durch die Kombination von Waschung und Desinfektion im Vergleich zur alleinigen Waschung (nach [20]).

tion) geführt hat als die alleinige Waschung ( $\bullet$ Abb.2). Dieses könnte zum einen ein distinkter Effekt des Ethanols sein, es könnte sich jedoch ebenfalls um einen unspezifischen Auswascheffekt von Detergentienmonomeren durch das Ethanol handeln. Daher führten wir eine Anschlusstestung mit der Kombination aus Händewaschung und alkoholischer Händedesinfektion sowie Händewaschung und anschließender Spülung mit Wasser durch. Die Irritation hier zeigte sich nun identisch, sodass der beobachtete Effekt am ehesten ein unspezifischer Auswascheffekt von Detergentienmonomeren durch das Ethanol darstellt [20]. Diese Studie untermauert jene Arbeiten, welche ebenfalls der alkoholischen Händedesinfektion eine deutlich bessere Hautverträglichkeit attestieren als der hygienischen Händewaschung [21,22]. In weiteren Untersuchungen konnte belegt werden, dass mit der Zugabe von hautpflegenden Inhaltsstoffen (wie z. B. Glycerin) die Verträglichkeit noch einmal deutlich gesteigert werden kann $[23,24]$.

Die Studien belegen eindrücklich, dass durch die alkoholische Händedesinfektion es nur zu einer geringgradigen Veränderung der epidermalen Barriere kommt, hingegen durch eine Händewaschung mit Detergentien die Barriere deutlich stärker geschädigt wird. Auch der austrocknende Effekt der Detergentienwaschung ist eher stärker als der der alkoholischen Händedesinfektion.

\section{Das Compliance-Problem \\ $\nabla$}

Jede Händehygiene-Maßnahme kann nur effektiv sein, wenn sie im Alltag auch korrekt angewendet wird. Auffallend ist, dass die Compliance Rate der korrekten Händedesinfektion mit < 50\% sehr gering ist [2]. Diese niedrige Compliance-Raten haben natürlich vielfältige Ursachen, wie z. B. die Anzahl der zur Verfügung stehenden Spender [25], die Zeitdauer der Anwendung [1], Arbeitsbelastung und Personalmangel [26,27] aber eben auch die Hautverträglichkeit der Anwendung [1,20]. Der guten Studienlage bezüglich der Hautverträglichkeit von alkoholischen Händedesinfektionsmitteln steht aber die skeptische Einschätzung des Pflegepersonals entgegen [16], was zu der insge-

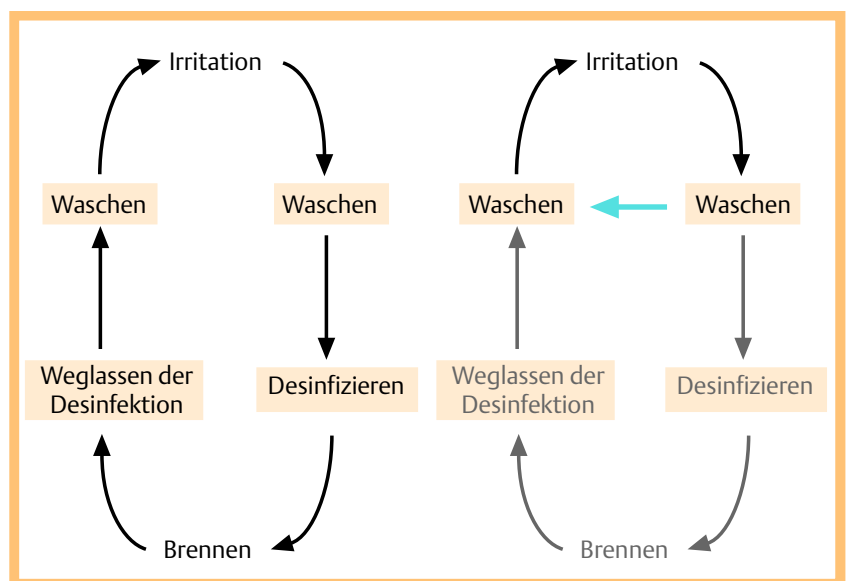

Abb. 3 Der circulus vitiosus: Aufgrund einer Barriereschädigung brennt die alkoholische Händedesinfektion (ohne jedoch die Haut weiter zu schädigen), der Anwender reduziert jedoch deswegen die Desinfektion und wäscht die Hände lieber (was auch nicht brennt). Dadurch verschlimmert sich jedoch die Irritation weiter.

samt geringen Compliance-Rate beitragen dürfte. Ein Grund dafür liegt sicherlich darin, dass alkoholische Händedesinfektionsmittel brennende Sensationen hervorrufen können [28]. Dieses Brennen tritt insbesondere auf klinisch wie subklinisch bereits veränderter Haut auf, insbesondere bei geschädigter Barriere. Der Alkohol kann hier an die sensiblen Nervenendigungen der Haut vordringen und diese reizen, welches der Anwender dann als Brennen verspürt. Reflexartig wird er die alkoholische Händedesinfektion nun für diese brennende Sensation verantwortlich machen, und das Händedesinfektionsmittel als „scharfes Präparat“ verurteilen. Da er nunmehr der Meinung ist, die alkoholische Händedesinfektion würde seiner Haut schaden, wird er diese zunehmend meiden und die Hände mehr waschen denn sie zu desinfizieren. Dieses führt jedoch kontinuierlich zu einer weiteren Verschlechterung seines Hautzustandes, sodass es von der ursprünglichen leichten Irritation eher zu einem stärkeren Handekzem fortschreiten kann $(\bullet$ Abb. 3) [28]. 
Diesem circulus vitiosus gilt es vorzubeugen. Sollte ein Mitarbeiter eine brennende Sensation nach der Anwendung von alkoholischen Händedesinfektionsmitteln verspüren, so muss ihm klar gemacht werden, dass diese Sensation ein Zeichen dafür ist, dass seine Haut bereits geschädigt ist. Die alkoholische Händedesinfektion wird nun diese Schädigung kaum verstärken, dient aber als Indikator der Intaktheit der Haut. Der Anwender sollte nunmehr eher die Händewaschung reduzieren, okklusive Tätigkeiten weitestgehend vermeiden und eine gute Behandlung der Haut mit Hautschutz- und Hautpflegecremes durchführen. Eine diesbezügliche Aufklärung, insbesondere bei Einführung von alkoholischen Händedesinfektionsmitteln ist essenziell für den Erfolg $[25,29,30]$.

Leider ist uns von klein auf der Gebrauch von Wasser und Seife viel eher in die Wiege gelegt worden als der von alkoholischen Händedesinfektionsmitteln. Haben wir das Gefühl, die Hände sind dreckig, so wird eher eine Händewaschung vorgenommen denn eine Händedesinfektion. Aus hygienischer Sicht ist zur Keimreduktion jedoch die alkoholische Händedesinfektion wesentlich effektiver denn das Händewaschen. Der Unterschied beträgt abhängig vom Alkohol (und je nachdem, ob man die transiente oder residente Flora betrachtet) zwischen 2 und 6 log-Stufen [31-33]. Es ist sogar so, dass durch die Händewaschung mindestens über 10 Minuten eine verstärkte Hydratation der obersten Hornschichten zustande kommt [34]. Mikroorganismen können sich in diesem „Hydratationsmantel“ eher verstecken und die alkoholische Händedesinfektion überleben. Bei einer anschließenden Händedesinfektion wird diese daher tendenziell weniger wirksam sein als auf einer nicht vorher gewaschenen Haut. Dieses führt zu recht einfachen Empfehlungen: vor der alkoholischen Händedesinfektion sollten die Hände routinemäßig nur dann gewaschen werden, wenn sie eine sichtbare Verschmutzung aufweisen $[35,36]$.

Neben der verständlichen Sorge um eine gesunde Haut des Pflegepersonals, ist die Qualität der Haut auch in Bezug auf nosokomiale Infektionen von essenzieller Bedeutung. Es ist mittlerweile unumstritten, dass die Keimbesiedlung der Haut bei Schädigung der Hautbarriere ansteigt. Die Bakteriendichte ist auf Handekzemen deutlich höher als auf intakter Haut, was zu einem eigenen Risikofaktor für nosokomiale Infektionen und der Verbreitung von multiresistenten Keimen führt [30,37,38].

\section{Primäre Prävention \\ $\nabla$}

Da vielfach die Angewohnheiten während des Jahre und Jahrzehnte dauernden Berufsalltages antrainiert und internalisiert sind, ist eine Änderung dieses Verhaltens sehr schwer. Das Verhalten kann in aller Regel nur dann effektiv verändert werden, wenn durch frühzeitige Schulungen das richtige Wissen zu einem richtigen Verhalten führt [7,39-41]. Hier sind Mechanismen der primären Prävention bereits während der Berufsausbildung angesagt. Dass diese primäre Prävention auch im Gesundheitsdienst greift, konnten wir in unserer Studie „Primary prevention in health care employee - Price“ deutlich zeigen [42]. Wir untersuchten in dieser prospektiven randomisierten Interventionsstudie 521 Pflegeschüler aus 14 Pflegeschulen (Krankenpflege, Altenpflege, Kinderkrankenpflege sowie Hebammen). Die Schüler wurden zur Hälfte einer regelmäßigen Schulung (2bis 3-mal pro Jahr) unterzogen, in denen ihnen Wissen über die Struktur und Funktion der Haut vermittelt wurde, die Mechanismen der Irritation erklärt wurden sowie Gegenmaßnahmen wie

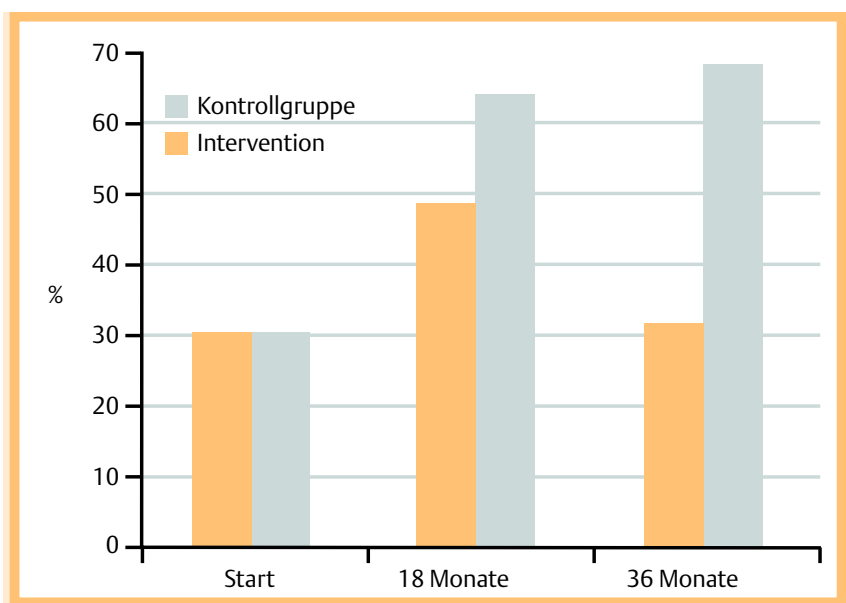

Abb. 4 Anteil der irritativen Hautveränderung (in \% aller Untersuchten) in der geschulten Kohorte (Intervention) und der Kontrollkohorte (nach [42]).

Hautschutz und Hautpflege erläutert wurden. Dieses geschah sowohl als Vortrag als auch in Form von Rollenspielen und praktischen Übungen. Aufgrund dieser intensiven Intervention bereits während der Ausbildung konnte nach den 3 Jahren Ausbildung bereits eine Halbierung der irritativen Hautveränderungen im Vergleich zur nicht geschulten Kontrollgruppe beobachtet werden ( $\bullet$ Abb.4). Das Risiko, irritative Hautveränderungen zu entwickeln, war in der Kontrollgruppe um das 4,7-Fache erhöht im Vergleich zur Schulungsgruppe. Der Erfolg konnte daran gemessen werden, dass in der geschulten Gruppe deutlich weniger die Hände gewaschen wurden und deutlich mehr alkoholische Händedesinfektionmittel verwandt wurden, die Hautpflege wurde intensiver durchgeführt, während Flächendesinfektion wurden mehr Handschuhe benutzt und auch während der arbeitsfreien Zeit wurde mehr Hautpflege und Hautschutz angewandt [42].

In der Konsequenz heißt dieses, dass der richtige Umgang mit Händehygiene (Händewaschen, alkoholische Händedesinfektion, Hautschutz und Hautpflege) bereits früh erlernt werden muss, um die Wahrscheinlichkeit der Entwicklung irritativer Hautveränderungen im Berufsleben möglichst gering zu halten.

\section{Abstract}

Cleanliness Turns into Illness - The Interconnection of Handhygiene and Hand Excema $\nabla$

Irritant skin changes and dermatitis on the hands of health care employees are quite common and belong to daily routine. Measures of hand disinfection are frequently accused of inducing these changes. Particularly the hand disinfection with alcohol based skin rubs is blamed to be responsible for their dry and scaly skin. Even allergic reactions to the hand rubs are assumed. In extensive testing, no allergic reactions to alcohols have been described so far. Only sensitization against other ingredients of alcohol based hand rubs have been found. The performed irritant patch testing as well as the in use tests of the alcohols have shown a remarkably good tolerance - far better than the one to hand washing with a detergent. The reason why many health care employees believe to have intolerance against alcohol based hand rubs is that they feel burning and stinging sensation after 
application of the product on hands with an impaired barrier. They accuse the alcohol based hand rub for this sensation and tend to use hand washing instead, which impairs their skin barrier more seriously. An educational teaching, most effectively during training, will help to avoid such behaviour and may help to maintain a good skin condition.

\section{Literatur}

1 Boyce JM, Pittet D. Guideline for Hand Hygiene in Health-Care Settings. Recommendations of the Healthcare Infection Control Practices Advisory Committee and the HIPAC/SHEA/APIC/IDSA Hand Hygiene Task Force. Am J Infect Control 2002; 30: 1 - 46

2 Pittet $D$. Compliance with hand disinfection and its impact on hospital-acquired infections. J Hosp Infect 2001; 48 (Suppl A): S40-S46

3 Schnuch A, Uter W, Geier J, Frosch PJ, Rustemeyer T. Contact allergies in healthcare workers. Results from the IVDK. Acta Derm Venereol 1998; 78: $358-363$

4 Kavli G, Angell E, Moseng D. Hospital employees and skin problems. Contact Dermatitis 1987; 17: 156-158

5 Smit HA, Burdorf A, Coenraads PJ. Prevalence of hand dermatitis in different occupations. Int J Epidemiol 1993; 22: 288 - 293

6 Nilsson E, Back 0 . The importance of anamnestic information of atopy, metal dermatitis and earlier hand eczema for the development of hand dermatitis in women in wet hospital work. Acta Derm Venereol 1986; 66: $45-50$

7 Stingeni L, Lapomarda V, Lisi P. Occupational hand dermatitis in hospital environments. Contact Dermatitis 1995; 33: $172-176$

8 Dickel H, Kuss O, Schmidt A, Kretz J, Diepgen TL. Importance of irritant contact dermatitis in occupational skin disease. Am J Clin Dermatol 2002; 3: 283-289

9 Dickel H, Kuss O, Blesius CR, Schmidt A, Diepgen TL. Report from the register of occupational skin diseases in northern Bavaria (BKH-N). Contact Dermatitis 2001; 44: 258-259

10 Dickel H, Kuss O, Blesius CR, Schmidt A, Diepgen TL. Occupational skin diseases in Northern Bavaria between 1990 and 1999: a populationbased study. Br J Dermatol 2001; 145: 453-462

11 Schwanitz HJ, Uter W. Interdigital dermatitis: sentinel skin damage in hairdressers. Br J Dermatol 2000; 142: 1011 - 1012

12 Kampf G. Händehygiene im Gesundheitswesen. Berlin: Springer-Verlag, 2002

13 Flyvholm MA, Bach B, Rose M, Jepsen KF. Self-reported hand eczema in a hospital population. Contact Dermatitis 2007; 57: 110-115

14 Pittet $D$. Improving compliance with hand hygiene in hospitals. Infect Control Hosp Epidemiol 2000; 21: 381 - 386

15 Pittet $D$. Improving adherence to hand hygiene practice: a multidisciplinary approach. Emerg Infect Dis 2001; 7: 234-240

16 Stutz N, Uter W, Spornraft-Ragaller P et al. Nurses perception of hand disinfection: alcohol-based hand rubs versus hygienic handwashing - a multicenter questionnaire study with additional patch testing by the german contact dermatitis research group (DKG). Br J Dermatol 2008; in press

17 Kampf G. Personal Communication regarding the amount of alcohol based hand rubs used per year in germany. Hamburg: Bode-Chemie, 2008

18 Okazawa H, Aihara M, Nagatani T, Nakajima H. Allergic contact dermatitis due to ethyl alcohol. Contact Dermatitis 1998; 38: 233

19 Ophaswongse S, Maibach HI. Alcohol dermatitis: allergic contact dermatitis and contact urticaria syndrome. A review. Contact Dermatitis 1994; 30: 1-6

20 Löffler H, Kampf G, Schmermund D, Maibach HI. How irritant is alcohol? Br J Dermatol 2007; 157: 74 - 81
21 Pedersen LK, Held E, Johansen JD, Agner T. Short-term effects of alcoholbased disinfectant and detergent on skin irritation. Contact Dermatitis 2005; $52: 82-87$

22 Pedersen LK, Held E, Johansen JD, Agner T. Less skin irritation from alcohol-based disinfectant than from detergent used for hand disinfection. Br J Dermatol 2005; 153: 1142 - 1146

23 Slotosch CM, Kampf G, Löffler H. Effects of disinfectants and detergents on skin irritation. Contact Dermatitis 2007: 57: 235-241

24 Kampf G, Wigger-Alberti W, Schoder V, Wilhelm KP. Emollients in a propanol-based hand rub can significantly decrease irritant contact dermatitis. Contact Dermatitis 2005; 53: 344- 349

25 Bischoff WE, Reynolds TM, Sessler CN et al. Handwashing compliance by health care workers: The impact of introducing an accessible, alcohol-based hand antiseptic. Arch Intern Med 2000; 160: 1017- 1021

26 Pessoa-Silva CL, Toscano CM, Moreira BM et al. Infection due to extended-spectrum beta-lactamase-producing Salmonella enterica subsp. enterica serotype infantis in a neonatal unit. J Pediatr 2002; 141: $381-387$

27 Vicca AF. Nursing staff workload as a determinant of methicillin-resistant Staphylococcus aureus spread in an adult intensive therapy unit. J Hosp Infect 1999; 43: 109- 113

28 Lübbe J, Ruffieux C, Perrenoud D. A stinging cause for preventive skin care. Lancet 2000; 356: $768-769$

29 Girard R, Amazian K, Fabry J. Better compliance and better tolerance in relation to a well-conducted introduction to rub-in hand disinfection. J Hosp Infect 2001; 47: 131 - 137

$30 \mathrm{Kampf}$, Löffler H. Dermatological aspects of a successful introduction and continuation of alcohol-based hand rubs for hygienic hand disinfection. J Hosp Infect 2003; 55: 1 - 7

31 Rotter ML. Arguments for alcoholic hand disinfection. J Hosp Infect 2001; 48 (Suppl A): S4-S8

32 Girou E, Loyeau S, Legrand P et al. Efficacy of handrubbing with alcohol based solution versus standard handwashing with antiseptic soap: randomised clinical trial. Bmj 2002; 325: 362

$33 \mathrm{Kampf}$ G, Kramer A. Epidemiologic background of hand hygiene and evaluation of the most important agents for scrubs and rubs. Clin Microbiol Rev 2004; 17: 863-893, table of contents

34 Hübner NO, Kampf G, Löffler H, Kramer A. Effect of a $1 \mathrm{~min}$ hand wash on the bactericidal efficacy of consecutive surgical hand disinfection with standard alcohols and on skin hydration. Int J Hyg Environ Health 2006; 209: 285- 291

35 Kramer A, Junger M, Kampf G. Hygienische und dermatologische Aspekte der Handedesinfektion und der prophylaktischen Hautantiseptik. Hautarzt 2005; 56: $743-751$

36 Hübner NO, Kampf G, Kamp P et al. Does a preceding hand wash and drying time after surgical hand disinfection influence the efficacy of a propanol-based hand rub? BMC Microbiol 2006; 6: 57

37 Larson E, Friedman C, Cohran J et al. Prevalence and correlates of skin damage on the hands of nurses. Heart Lung 1997; 26: 404-412

38 Ojajarvi J, Makela P, Rantasalo I. Failure of hand disinfection with frequent hand washing: a need for prolonged field studies. J Hyg (Lond) 1977; 79: 107 - 119

39 Elsner P. Irritant dermatitis in the workplace. Dermatol Clin 1994; 12 : $461-467$

40 Wigger-Alberti $W$, Elsner P. Preventive measures in contact dermatitis. Clin Dermatol 1997; 15: 661 - 665

41 Wigger-Alberti W, Maraffio B, Wernli M, Elsner P. Training workers at risk for occupational contact dermatitis in the application of protective creams: efficacy of a fluorescence technique. Dermatology 1997; 195: $129-133$

42 Löffler H, Bruckner T, Diepgen T, Effendy I. Primary prevention in health care employees: a prospective intervention study with a 3-year training period. Contact Dermatitis 2006; 54: 202 -209 